\title{
The mass spectrum of double heavy baryons in new potential quark models
}

\author{
Vladimir Kovalenko ${ }^{1, a}$ and Andrei Puchkov ${ }^{1}$ \\ ${ }^{1}$ Saint Petersburg State University, Russia
}

\begin{abstract}
A new approach to study the mass spectrum of double heavy baryons $\left(Q Q^{\prime} q\right)$ containing strange and charmed quarks is proposed. It is based on the separation of variables in the Schrodinger equation in the prolate spheroidal coordinates. Two nonrelativistic potential models are considered. In the first model, the interaction potential of the quarks is the sum of the Coulomb and non-spherically symmetrical linear confinement potential. In the second model it is assumed that the quark confinement provided by a spherically symmetric harmonic oscillator potential. In both models the mass spectrum is calculated, and a comparison with previous results from other models is performed.
\end{abstract}

\section{Motivation}

The study the properties of baryons containing two heavy quarks is of considerable interest for the solution the confinement problem in QCD. It is related to the fact that the masses of heavy quarks $m_{Q}$ define a new energy scale exceeding the the strong interaction scale $\Lambda_{Q C D}$ :

$$
m_{Q} \gg m_{q}, \quad R \Lambda_{Q C D} \ll 1, \quad \Lambda_{Q C D} \ll m_{Q} .
$$

Here $R$ is the distance between two heavy quarks, $m_{q}$ is the mass of light quark. Thus, in theory, there is a small parameter $m_{q} / m_{Q}$, which can be used for the application of the perturbative approach.

The baryons containing two heavy quarks are becoming the subject of extensive theoretical study in recent years. In 2002 the Selex Collaboration observed the baryon $\Xi_{c c}^{+}(c c d)$ in the decay [1]

$$
\Xi_{c c}^{+} \rightarrow \Lambda_{c}^{+} K^{-} \pi^{+}
$$

and later [2]

$$
\Xi_{c c}^{+} \rightarrow p D^{+} K^{-}
$$

The mass of the state was measured to be:

$$
M_{\Xi_{c c}^{+}}=3518.9 \pm 0.9 \mathrm{MeV} .
$$

\footnotetext{
a e-mail: v.kovalenko@spbu.ru
} 


\section{Formalism}

Double heavy baryons can be studied by potential models in two different ways. The first one consists in studying the three body problem, in which the potential term is the sum of the interactions between each pair of quarks [3-5]. The second one consists in studying the two body problem, in which one quark interacts with a bound state of the other two quarks [6]. The double heave baryon mass is equal to

$$
M=m_{Q}+m_{Q^{\prime}}+m_{q}+E_{Q Q^{\prime} q}
$$

where $E_{Q Q^{\prime} q}$ the binding energy.

The double heavy baryons may be considered as an analog of the hydrogen molecular ion $\mathrm{H}_{2}^{+}$, which has been treated successfully in the Born-Oppenheimer approximation. In this approximation the wave function is split into heavy- and light-quark degree of freedom:

$$
\Psi(R, r)=\sum_{n} a_{n} \phi_{n}(R) \psi_{n}(R, r)
$$

where $R$ is the distance between two heavy quark and $r$ is the distance between the light quark and center of mass of diquark. The light-quark wave function $\psi_{n}(R, r)$ and the its energy term $E_{q}(R)$ can be found from Schrödinger equation

$$
\Delta \psi+2 m_{q}\left(E_{q}(R)-V\right) \psi=0 .
$$

Note that in the system of units we use $\hbar=1$.

Prolate spheroidal coordinates are determined by the following relations [7]:

$$
\begin{gathered}
\xi=\frac{r_{1}+r_{2}}{R}, \quad \eta=\frac{r_{1}-r_{2}}{R}, \quad \varphi=\arctan \left(\frac{y}{x}\right), \\
+1 \leqslant \xi \leqslant \infty, \quad-1 \leqslant \eta \leqslant+1, \quad 0 \leqslant \varphi \leqslant 2 \pi,
\end{gathered}
$$

where $r_{1}$ and $r_{2}$ - distances between heavy quarks and light.

\section{First model}

The first model of double heavy baryons assumes that the potential $V$ in (3) consist of Coulomb and confinement terms and is equal to

$$
V_{1}=V_{\text {Coul }}+V_{\text {conf }}=-\frac{\left(Z_{1}+Z_{2}\right) \xi+\left(Z_{2}-Z_{1}\right) \eta}{R\left(\xi^{2}-\eta^{2}\right)}+\frac{\beta R \xi\left(\xi^{2}-1\right)}{\left(\xi^{2}-\eta^{2}\right)}-\frac{4}{3} V_{0},
$$

Obviously, the potential (6) for large $\xi$ behaves as:

$$
V_{1} \underset{\xi \rightarrow \infty}{\longrightarrow} \beta R \xi \sim r,
$$

satisfying thus the correct linear asymptotic behavior. This potential (6) is shown in the Figure 1.

Let us represent the wave function $\psi_{j}$, corresponding to the term $E_{j}(R)$, in the form of

$$
\psi_{j}=\psi_{k q m}(\xi, \eta, \varphi ; R)=N_{k q m}(R) \frac{X_{m k}(\xi ; R)}{\sqrt{\xi^{2}-1}} \frac{Y_{m q}(\eta ; R)}{\sqrt{1-\eta^{2}}} e^{i m \varphi},
$$




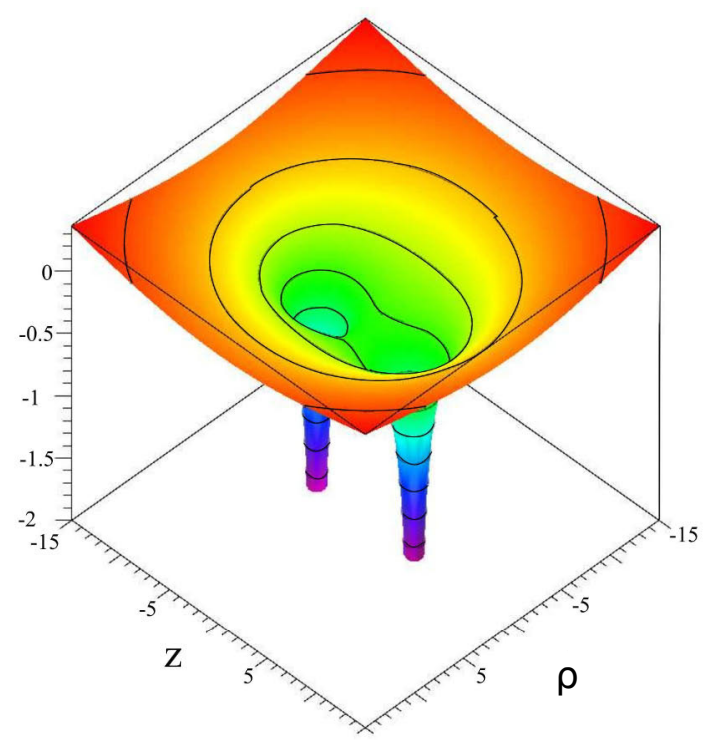

Figure 1. Potential of the interaction between light and heavy quarks. Here $\rho$ and $z$ are cylindrical coordinates.

where the multiindex $j=\{k q m\}$ denotes the quantum number set in which $k$ and $q$ coincide with the numbers of zeros of the corresponding functions in the variables $\xi$ and $\eta$, and the number $m$ takes on the values $0, \pm 1, \pm 2, \ldots$. The normalization constant $N_{k q m}(R)$ is determined by the condition

$$
\int_{V} \psi_{k q m}^{*}(\xi, \eta, \varphi ; R) \psi_{k^{\prime} q^{\prime} m^{\prime}}(\xi, \eta, \varphi ; R) d V=\delta_{k k^{\prime}} \delta_{q q^{\prime}} \delta_{m m^{\prime}}
$$

where $d V=\frac{R^{3}}{8}\left(\xi^{2}-\eta^{2}\right) d \xi d \eta d \varphi-$ is a volume element in the prolate spheroidal coordinates.

After substituting (6) and (8) into (3) we obtain the ordinary differential system

$$
\begin{gathered}
\frac{d^{2} X_{m k}(\xi ; R)}{d \xi^{2}}+\left(m_{q}\left[-p^{2}-\beta R^{3} \xi+\frac{a \xi-\lambda_{m k}^{(\xi)}}{\xi^{2}-1}\right]+\frac{m^{2}-1}{\left(\xi^{2}-1\right)^{2}}\right) X_{m k}(\xi ; R)=0, \\
\frac{d^{2} Y_{m q}(\eta ; R)}{d \eta^{2}}+\left(m_{q}\left[-p^{2}+\frac{b \eta+\lambda_{m q}^{(\eta)}}{1-\eta^{2}}\right]-\frac{m^{2}-1}{\left(1-\eta^{2}\right)^{2}}\right) Y_{m q}(\eta ; R)=0 .
\end{gathered}
$$

Here

$$
p_{j}^{2}=-\frac{R^{2}}{2}\left(E_{j}+\frac{4}{3} V_{0}\right), \quad a=\left(Z_{1}+Z_{2}\right) R, \quad b=\left(Z_{2}-Z_{1}\right) R,
$$

$p$ has the meaning of the energy parameter; $a, b$ are the charge parameters; $\lambda_{m k}^{(\xi)}=\lambda_{m k}^{(\xi)}(p, a)$, $\lambda_{m q}^{(\eta)}=\lambda_{m q}^{(\eta)}(p, b)$ are the separation constants.

The equations (9) and (10) are supplemented by the boundary conditions:

$$
X_{m k}(+1 ; R)=0, \quad\left|X_{m k}(\xi ; R)\right| \underset{\xi \rightarrow \infty}{\longrightarrow} 0, \quad\left|Y_{m q}( \pm 1 ; R)\right|<\infty .
$$


Thus the equations (9) - (12) form the boundary value problems that must be solved simultaneously, and the energy spectrum can be obtained from the condition

$$
\lambda_{m k}^{(\xi)}(p, a)=\lambda_{m q}^{(\eta)}(p, b) .
$$

Let's find the binding energy of the double heavy baryon $E_{Q Q^{\prime} q}$. After separation of the angular variables and substitution

$$
\phi_{n l}(R)=\frac{F_{n l}(R)}{R}
$$

for the eigenfunctions, we obtain

$$
\frac{d^{2} F_{n l}(R)}{d R^{2}}+\left[2 M_{\text {red }}\left(E_{Q Q^{\prime} q}-E_{q}(R)+\frac{2 \alpha_{s}}{3 R}-\beta R\right)-\frac{l(l+1)}{R^{2}}\right] F_{n l}(R)=0 .
$$

Equation (14), supplemented by the boundary conditions

$$
F_{n l}(0)=0, \quad\left|F_{n l}(R)\right| \underset{R \rightarrow \infty}{\longrightarrow} 0
$$

forms a boundary value problem, by solving which we find $E_{Q Q^{\prime} q}$. The equations (9), (10), (14) are solved numerically.

\section{Second model}

In the second model, inspired by [3], the potential is taken as:

$$
V_{2}=-\frac{Z_{1}}{r_{1}}-\frac{Z_{2}}{r_{2}}+\omega^{2}\left(r_{1}^{2}+r_{2}^{2}\right)-\frac{4}{3} V_{0}
$$

In prolate spheroidal coordinates it is given by:

$$
V_{2}=V_{\text {Coul }}+V_{\text {con }}=-\frac{a(\xi)+b(\eta)}{R^{2}\left(\xi^{2}-\eta^{2}\right)}-\frac{\omega^{2} R^{2}}{2}-\frac{4}{3} V_{0},
$$

where

$$
\begin{aligned}
& a(\xi)=\left(Z_{1}+Z_{2}\right) R \xi-\frac{\omega^{2} R^{4}}{4} \xi^{2}\left(\xi^{2}-1\right), \\
& b(\eta)=\left(Z_{1}-Z_{2}\right) R \xi-\frac{\omega^{2} R^{4}}{4} \eta^{2}\left(\eta^{2}-1\right),
\end{aligned}
$$

Obviously, the potential (16) for large $\xi$

$$
V_{2} \underset{\xi \rightarrow \pm \infty}{\longrightarrow} \omega^{2} R^{4} \xi^{2} \sim r^{2},
$$

which differs from linear asymptotic behavior (7).

The Schrödinger equation with potential (16) admits a separation of variables. This allows us to obtain the ordinary differential system similar to (9)-(10):

$$
\begin{aligned}
& \frac{d^{2} X_{m k}(\xi ; R)}{d \xi^{2}}+\left(m_{q}\left[-p^{2}+\frac{a(\xi)-\lambda_{m k}^{(\xi)}}{\xi^{2}-1}\right]+\frac{m^{2}-1}{\left(\xi^{2}-1\right)^{2}}\right) X_{m k}(\xi ; R)=0, \\
& \frac{d^{2} Y_{m q}(\eta ; R)}{d \eta^{2}}+\left(m_{q}\left[-p^{2}+\frac{b(\eta)+\lambda_{m q}^{(\eta)}}{1-\eta^{2}}\right]-\frac{m^{2}-1}{\left(1-\eta^{2}\right)^{2}}\right) Y_{m q}(\eta ; R)=0 .
\end{aligned}
$$

The obtained boundary problems are solved in a similar way as for Model 1 . 


\section{Parameters fixation}

In the proposed models we consider only the case where both heavy quarks have the same flavor, i.e.

$$
Z_{1}=Z_{2}=\frac{2}{3} \alpha_{s}, \text { where } \alpha_{s}=0.39 .
$$

In the Model 1 , the tension parameter is taken as $\beta=0.116 \mathrm{GeV}^{2}$ to provide better agreement with the previous calculations. The other parameters are taken as in [3]: $\omega^{2}=0.174 \mathrm{GeV}^{3}$, the quark masses $m_{c}=1.486 \mathrm{GeV}, m_{b}=4.88 \mathrm{GeV}$ and $m_{q}=0.385 \mathrm{GeV}$; the constant term in the potential $V_{0}=0.05 \mathrm{GeV}$.

\section{Calculation of the observables}

The computed masses of the double heavy baryons with different combinations of the diquark and light quark bound states are listed in table. Also, it was assumed that the diquark is in the ground state.

Table 1. Masses of the double heavy baryons computed in the models and compared to known results

\begin{tabular}{|c|c|c|c|c|}
\hline State & Model 1 & Model 2 & Ref. [4] & Ref. [5] \\
\hline \multicolumn{5}{|c|}{$\Xi_{c c}, \mathrm{GeV}$} \\
\hline$(1 S 1 s) \frac{1}{2}^{+}$ & 3.581 & 3.661 & 3.520 & 3.685 \\
\hline$(2 S 1 s) \frac{1}{2}^{+}$ & 3.660 & 3.730 & 3.784 & 4.079 \\
\hline$(3 S 1 s) \frac{1}{2}^{+}$ & 3.672 & 3.816 & - & 4.159 \\
\hline \multicolumn{5}{|c|}{$\Xi_{b b}, \mathrm{GeV}$} \\
\hline$(1 S 1 s) \frac{1}{2}^{+}$ & 10.060 & 9.89 & - & 10.314 \\
\hline$(2 S 1 s) \frac{1}{2}^{+}$ & 10.178 & 9.911 & - & 10.571 \\
\hline$(3 S 1 s) \frac{1}{2}^{+}$ & 10.210 & 9.939 & - & 10.612 \\
\hline
\end{tabular}

\section{Conclusion}

We considered two non-relativistic potential models with separation of variables in prolate spheroidal coordinates. In the first model the confinement potential is not spherically symmetrical but has correct asymptotic behavior. In the second model the potential obey spherical symmetry but too fast grows at infinity. Despite the fact that the numerical calculations were carried out selectively and were of evaluative nature, it was shown that both models can successfully describe the experimental data and require further development.

\section{Acknowledgements}

This work is supported by the Russian Science Foundation (grant No. 16-12-10176). 


\section{References}

[1] M. Mattson et al. (SELEX Collaboration), Phys. Rev. Lett. 89, 11200 (2002).

[2] A. Ocherashvili et al. (SELEX Collaboration), Phys. Lett. B 628, 18 (2005).

[3] D. U. Matrasulov, M. M. Musakhanov, and T. Morii, Phys. Rev. C 61, 045204 (2000).

[4] A. Majethiya, B. Patel and P. C. Vinodkumar, Chinese. Phys. C 34, 1399 (2010).

[5] T. Yoshida, E. Hiyama, A. Hosaska, M. Oka and K. Sadato, Phys. Rev. D 92, 114029 (2015).

[6] V. V. Kiselev and A. K. Likhoded, Phys. Usp. 45, 455 (2002) [Usp. Fiz. Nauk 172, 497 (2002)].

[7] I. V. Komarov, L. I. Ponomarev and S. Yu. Slavyanov, Spheroidal and Coulomb Spheroidal Functions (Nauka, Moscow, 1976).

[8] A. M. Puchkov, A. V. Kozedub, AIP Conference Proceedings 1701, 100014 (2016); doi: $10.1063 / 1.4938723$. 